\title{
Internal Structure Quality Control of Solid Pharmaceuticals. A Comparative Study
}

\author{
Imre Silvia1 ${ }^{\star}$, Haidu Timea1, Ponta Oana², Vancea Szende1, Vari Camil-Eugen¹, Tero-Vescan Amelia1 \\ 1 University of Medicine and Pharmacy Tîrgu Mureș, Faculty of Pharmacy, Romania \\ 2 "Babeș-Bolyai" University, Faculty of Physics \& Interdisciplinary Research Institute on Bio-Nano-Sciences, Cluj-Napoca, Romania
}

Objective: The aim of the study was a comparative investigation by spectral and thermal analysis in order to asses a number of characteristics of different varieties ofrawmaterials of ursodeoxycholic acid and ibuprofen. The different dissolution behavior of two ursodeoxycholic acid pharmaceutical product by crystallinity pattern was investigated. Methods: Raw materials of ursodeoxycholic acid and ibuprofen were used. IR spectroscopy, differential scanning calorimetry and X-Ray Diffraction Analysis were applied. Results: The results show no crystallinitydifferences for different batches of the tested drugs. No solid solid transition was proved during sample preparation for transmission IR analysis. Conclusions: A combination of two more affordabletests by IR spectrometry and differential scanning calorimetry lead to the same results as $\mathrm{X}$-Ray diffraction analysis for crystallinity similarity assessment of the studied substances. The dissolution differences of test drugs were not related to the polymorphism of the raw materials.

Keywords: IR spectroscopy, DSC, X-Ray Diffraction Analysis (XRD), ibuprofen, ursodeoxycholic acid

Received: 12 August 2015 / Accepted: 05 September 2015

\section{Introduction}

In the pharmaceutical world the fact that active substances can exist in more than one crystal form (polymorph) can be considered today as a common knowledge. Each polymorph has different physical and physico-chemical properties in solid state such as crystal form, colour, dissolution rate, melting point, etc. In case of oral administration of solid pharmaceutical forms, one of the factors that influence the absorption of drug and therefore its therapeutic quality, is dissolution which is often strongly related to the polymorphism property. The differences between the physical properties of polymorphic systems are very important in developing a drug because the behavior of polymorphs can lead to implications in manufacturing, formulation and bioavailability.

In general, drug polymorphism research follows two main strategies: obtaining, characterisation and stability investigation of the pure substance and stability studies under pharmaceutical formulation conditions. The polymorphs stability relates to solid solid transition and it has to be divided further in stability during processing (technological or quality control processes) and long term stability under storage conditions. Few aspects related to polymorphs analysis in current pharmaceutical practice are given below.

It is a prerequisite now that quality control of solid pharmaceutical substances used for solid pharmaceutical formulations includes internal structure investigation in order to get the corresponding polymorph or amorph state. The analytical methods used for this reason include thermal analysis, X-ray diffraction spectrometry (XRD), infrared

* Correspondence to: Silvia Imre

E-mail: silviaimre@gmail.com
(IR) solid state spectrometry, Raman spectrometry, nuclear magnetic resonance (NMR) spectrometry etc. However different aspects have to take into consideration such as the sensibility and specificity of the analytical method for polymorhism investigation, polymorph conversion during sample preparation for analysis and so on. As an example, grinding and compressing before IR analyis could generate solid-solid transition.

Storage condition of pharmaceuticals can influence their crystallinity. Therefore the stability of a given polymorphic structure under storage conditions has to be very well understand when decision after the quality control results analysis has to be done.

The influence of salts formulation of drugs is another issue which has to be take into consideration for polymorphism investigation. The salt formation modifies the chemical properties of an active pharmaceutical ingredient such as log $\mathrm{P}$, pKa or melting point and, consequently, the parent drug behavior in the body due to the modifications of drug solubility, dissolution and chemical stability $[1$ Different pharmaceutical companies changed the nature of the counter ion for the same drug in order to treat different indications or alter the drug's pharmacokinetics. This practice is often encounterd for poor soluble acidic drugs. Ibuprofen is an example for which sodium or alkyl amine salts are reported.

The aim of the study was to highlight specific differences in crystalline structure between different batches with the same chemical nature of two acidic structures, ursodeoxcholic acid (UDO) and ibuprofen (IB), respectively (Figure 1). Crystalline pattern is influenced by the versatility of hydrogen bond formation, in particular carboxyl compounds being more susceptible due to the possibility of dimer or salt formation [2-3]. 


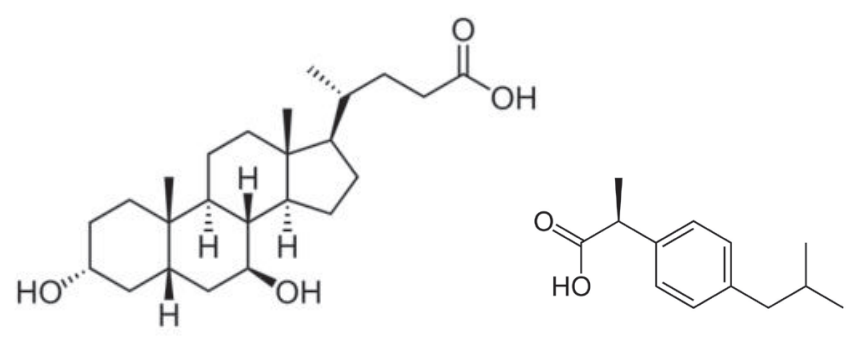

Fig. 1. Ursodeoxycholic acid (I) and ibuprofen (II) structures

The steroids belong to the general gonan chemical class with multiple biological effects, having endogeneous or nonendogeneous origin. There are more than 60 years of polymorphism research on steroid structure like substances, this property being often encountered in this chemical class. Ursodeoxycholic acid has a steroid structure with a carboxyl acidic group and it has an interesting behavior. It becomes X-ray amorphous following grinding or milling as indomethacin, an acidic compound, or digoxin, a substance with a gonan core [4-5].

Ibuprofen is a nonsteroidal anti-inflammatory drug (NSAID) derivative of propionic acid which was intensively studied not only from pharmacological point of view but also for pharmaceutical formulations improvement, including polymorphism investigations [6-9].

DSC and IR spectrometry were chosen being common analysis technique in quality control laboratory and XRD spectrometry was performed as reference analysis. Comparative analysis of several batches of ursodeoxycholic acid was started in order to find the cause of various dissolution profiles of pharmaceutical products of the same type but obtained with different raw materials of active substance. The identification of the studied samples was done by IR spectroscopy on the basis of pharmacopoeial previsions by electronic comparison with reference spectra to highlight the advantages and disadvantages of this method. In addition, we wanted to check whether in the case of the studied substances crystalline transformations may occur during the compression into potassium bromide tablets for analysis by IR spectrometry by comparison the results with those obtained through attenuated total reflectance IR analysis.

\section{Methods}

\section{Materials}

Three different batches of ursodeoxycholic acid samples (UDO-P1, UDO-P2, UDO-P3) and two different samples of ibuprofen raw materials (IB 38 and IB 40) were available from the different manufacturers. For sample preparation to FTIR analysis, potassium bromide for spectroscopy produced by Merck was used.

\section{Instruments}

IR analysis was carried out on a Nicolet 380 FTIR spectrometer from Thermo Electron Corporation using Omnic software. ATR-IR analysis was performend on a 470 Plus spectrometer by Jasco using the Spectra Manager software. Differential scanning calorimetry (DSC) was carried out on a DSC 60 produced by Shimadzu Corp. The diffraction analysis was performed with a Shimadzu XRD—6000 diffractometer.

Samples for IR analysis were prepared in the following way: for transmittance analysis, $10 \mathrm{mg}$ sample of pure substance was mixed with $100 \mathrm{mg}$ of dried potassium bromide for spectroscopy (Merck) and compressed into tablets with a hand press; for reflectance analysis, an appropriate amount of sample material was introduced in the ATR device's slot. Recording of IR spectra was made in the 4000-400 $\mathrm{cm}^{-1}$ interval at $4 \mathrm{~cm}^{-1}$ resolution.

For DSC analysis 2-6 mg sample of pure substance was introduced in a capsule and pressed into tablets. The temperature range was $30-250^{\circ} \mathrm{C}$, for ursodeoxycholic acid, and $25-100^{\circ} \mathrm{C}$, for ibuprofen. The temperature rate was $5^{0} \mathrm{C} / \mathrm{min}$.

The diffraction analysis was performed by using $\mathrm{Cu}$ $\mathrm{Ka}(\mathrm{k}=1.5405 \mathrm{~A})$ radiation, at a scan speed of $2 \mathrm{gr} / \mathrm{min}$.

Dissolution tests were accomplished following the previsions of Pharmacopoeia.

\section{Results and discussions}

X-ray diffractometry is the best technique for the study of polymorphs and solvatomorphs, by single-crystal $\mathrm{x}$-ray diffraction, not suitable for routine analysis, or by X-ray powder diffraction which is more useful. However the costs for equipment aquisition and staff training keep the generic pharmaceutical manufacturer far from this type of analysis. Thus, FTIR spectra and thermal analysis are often used to evaluate the type of polymorphism that exists in a drug substance and there are common determinations in pharmaceutical industry, being aware of their limitations [10].

Taking in account to these facts, the quality control study started with structure identification of the raw materials. According to official previsions [11-12], identification of a raw material by IR spectrometry can be made by: comparison with a spectrum of reference obtained on a substance in identical conditions; comparison with a reference spectrum from a database; highlighting intense characteristic vibration bands, oftenassigned to those atomic groups on which's level degradation reactions can be induced relatively easily.

Considering the comparative representation of the spectra of different batches of raw materials we can claim that identification by IR spectrometry of the analysed samples of UDO and IB raw materials allowed the confirmation of structures by comparison with reference spectra available in the electronic library of the equipment for IB and on paper print for UDO. The electronic spectral comparison method by assigning a score or similarity factor, available in Omnic software, is suitable as long as the spectra compensation of false contribution of $\mathrm{CO}_{2}$ and water vapours from the atmosphere are corrected. Otherwise, using only the 
similarity factor, we could falsely infirm the structural identity. With no atmosphere interferences compensation, ibuprofen obtained a similarity score over $93 \%$. On the other hand, in case of ursodeoxycholic acid, without electronic reference spectrum, the $56 \%$ similarity score to cholic acid indicates it's affiliation to the bile acids structural class.

Next, starting from the dissolution data, we decided to test whether the investigated raw materials, used to obtain different batches of ursodeoxycholic acid test pharmaceuticals, belong or not to the same crystalline or amorphous class because of the major behavioral differences observed at dissolution test at $\mathrm{pH} 8$ (Figure 2). The literature con-

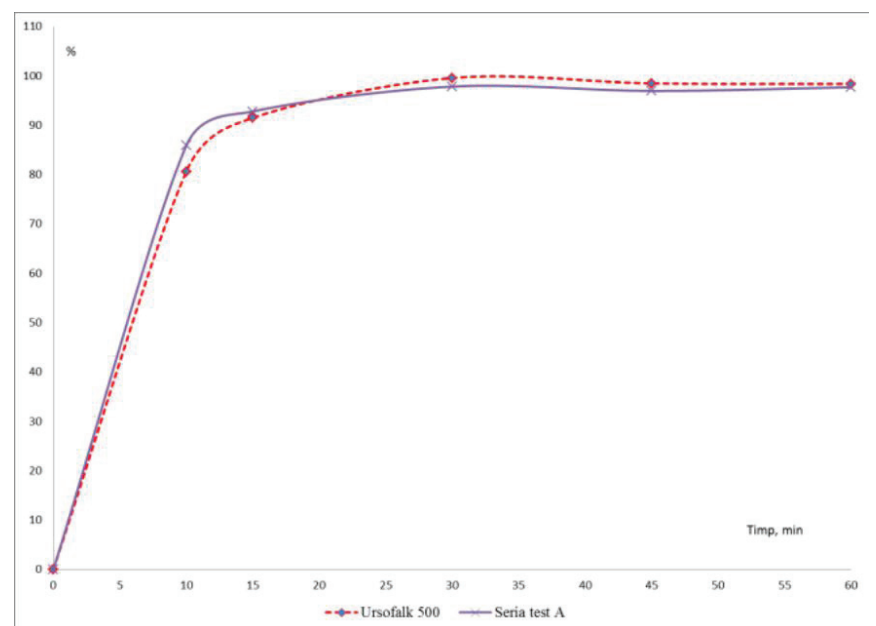

tains studies which, through thermal analysis, highlight the fact that the amorphous structure of ursodeoxycholic acid may be assigned to several states with similar solubility but with different forms of solid particles [13].

As shown by IR spectra (Figure 3) and XRD spectra (Figure 4), the three raw materials of ursodeoxycholic acid show the same spectral pattern which indicates identical crystalline arrangements. The samples had the same endothermic peak at $206{ }^{\circ} \mathrm{C}$ in DSC curves. In conclusion, the differences of dissolution behavior are not due to different states of internal arrangement of the molecules in the solid powder.

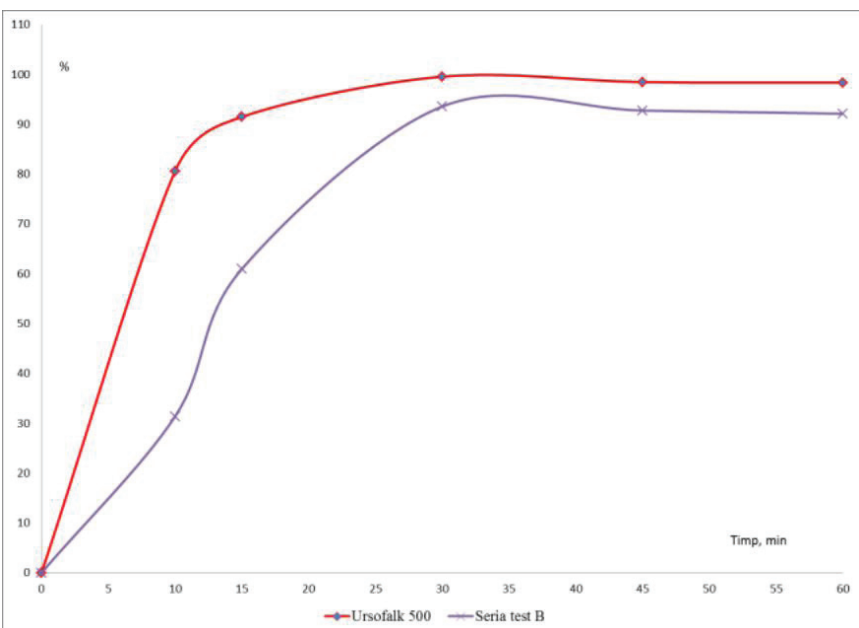

Fig. 2. Comparative dissolution curves of the two tested batches of pharmaceutical products versus reference product. Similarity factors are 77,4 and 28,9 , respectively

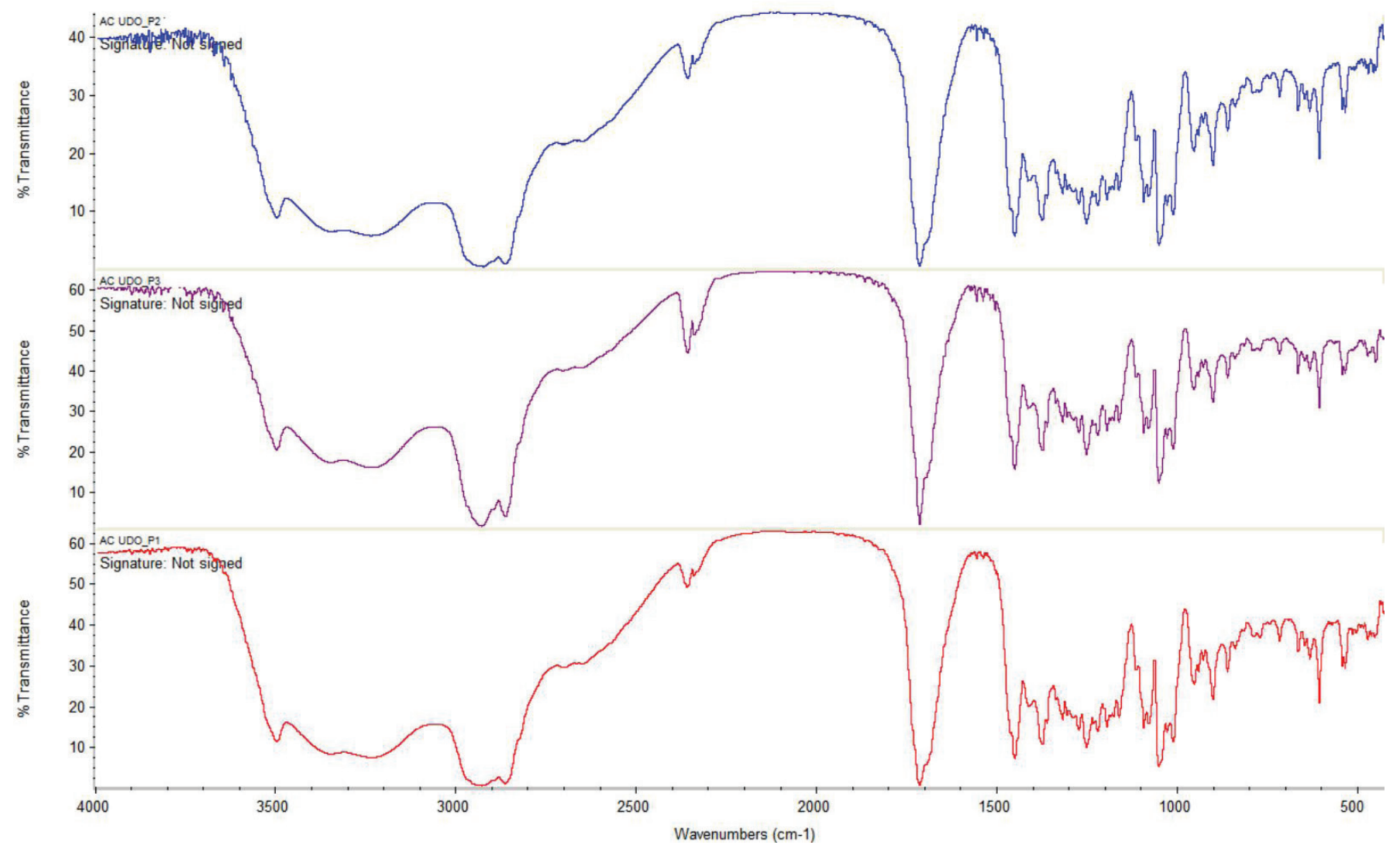

Fig. 3. IR spectra of the three batches of ursodeoxycholic acid UDO_P2, UDO_P3, UDO_P1 (from top to bottom) 

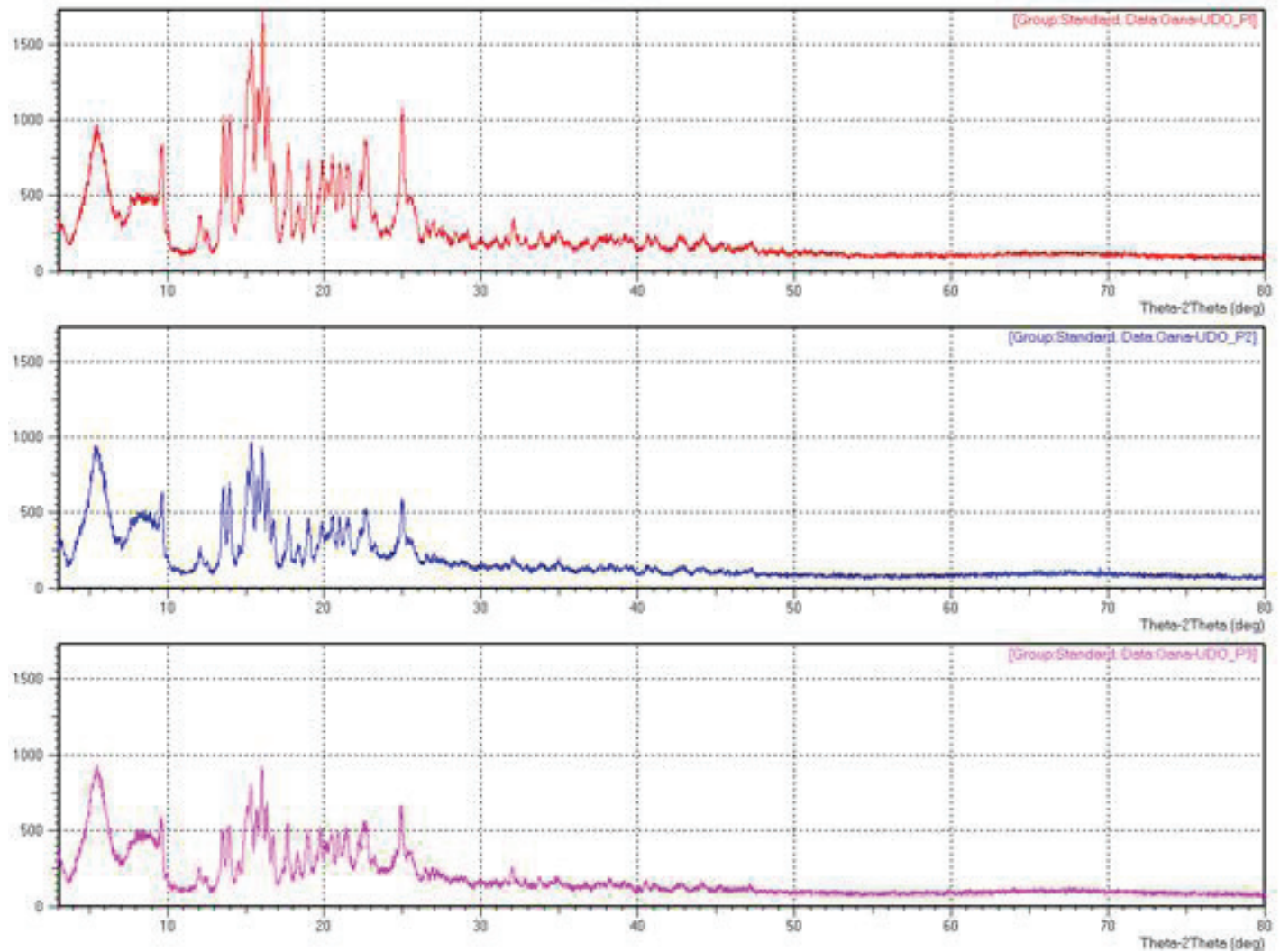

Fig. 4. XRD patterns of the three ursodeoxycholic acid samples UDO P1, UDO P2, UDO P3 (from top to bottom)
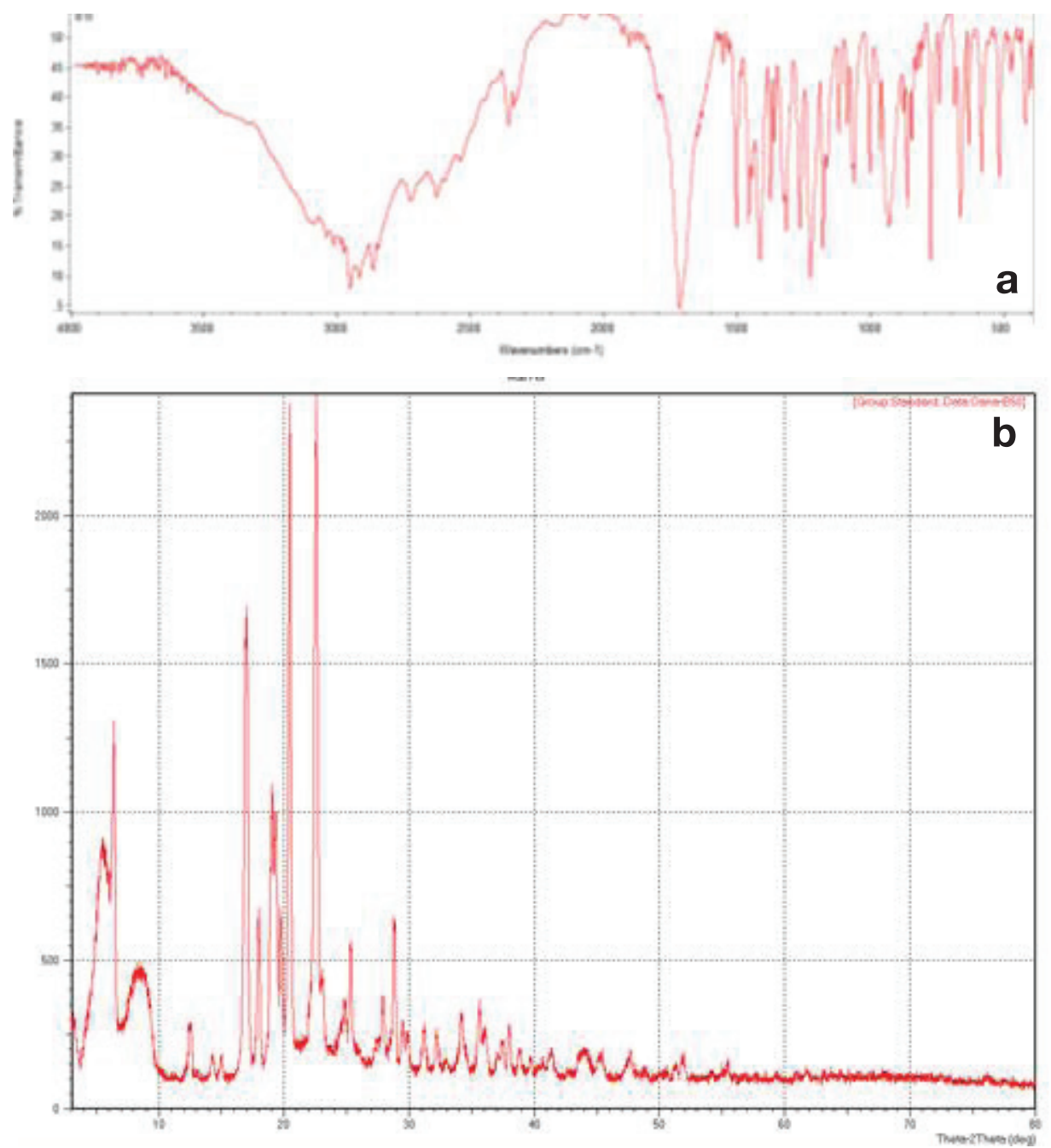

Fig. 5. a - IR and b - XRD pattern of ibuprofen raw material sample IB 50 
Proceeding in the same way we can conclude that the two batches of ibuprofen have the same polymorphic form, with identical IR, XRD spectra (Figure 5) and the same endothermic peak at $77^{\circ} \mathrm{C}$ in DSC curves.

Regarding the possible influence of sample preparation for IR analysis of polymorphs, the recorded IR spectra of studied raw materials by the method of $\mathrm{KBr}$ compression and also by direct analysis of the powder by attenuated to- tal reflectance show that there is no phase transition of the internal state of the solid powder during sample preparation by trituration and compressing (Figure 6). Possible differences for ursodeoxycholic acid were expected because it was proved that by milling processessolid transition occurs with different proportion of amorphous state depending on milling time [5].

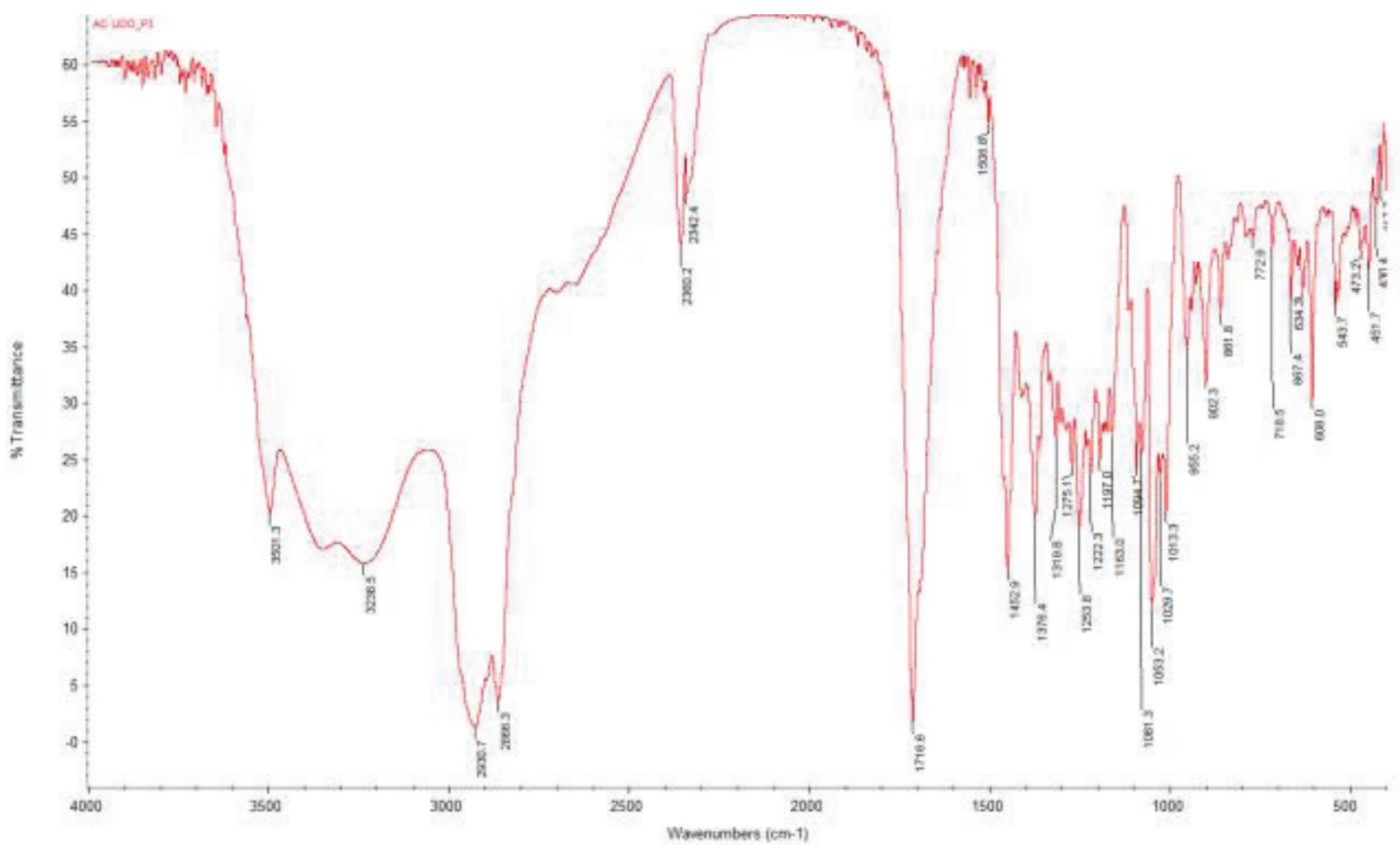

a

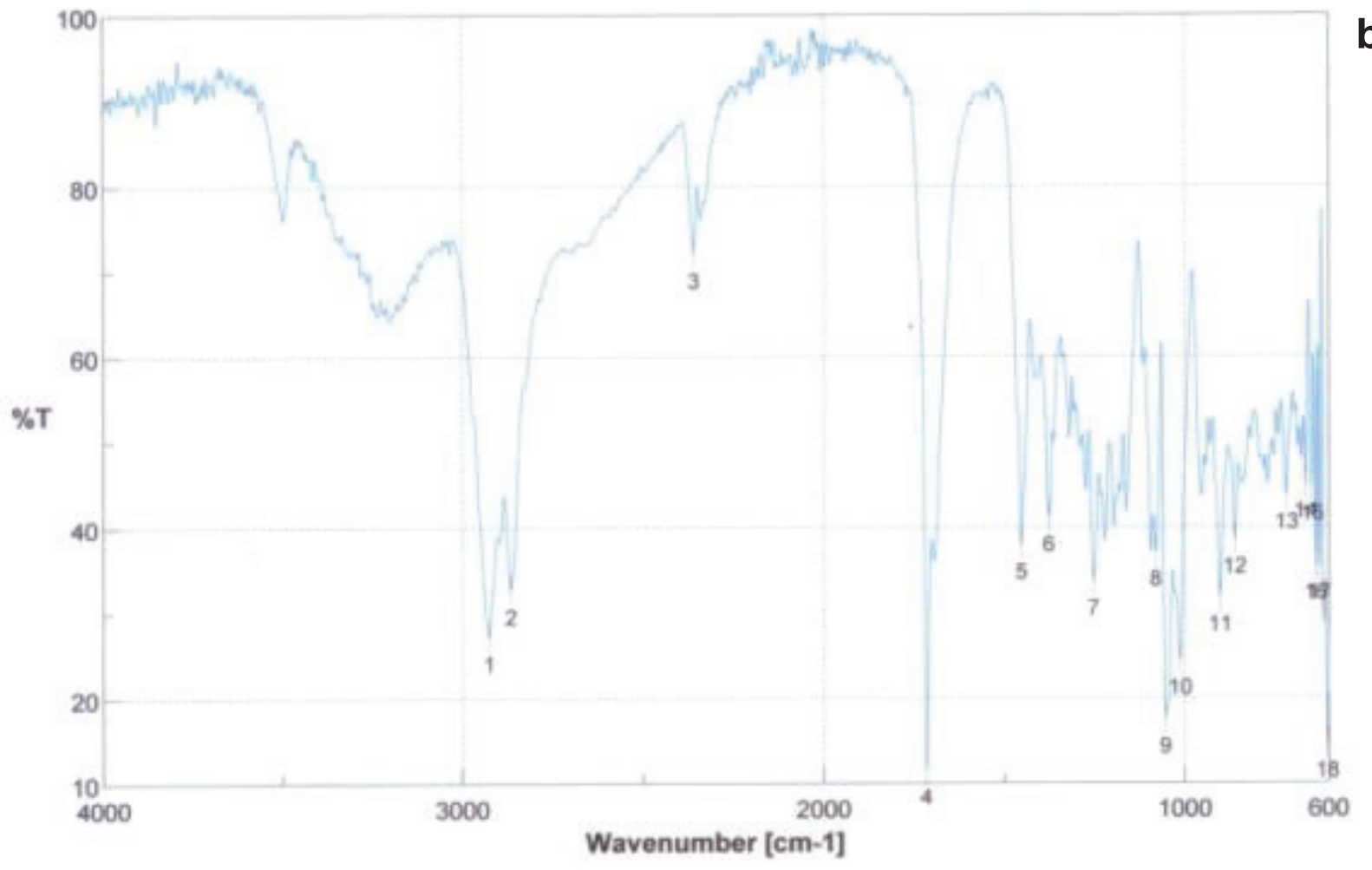

Fig. 6. IR spectra of ursodeoxycholic acid sample UDO P3: a - KBr sample preparation; $b$ - ATR analysis 


\section{Conclusions}

The similarity of the internal structure of the tested batches with the same substance was revealed by two more affordable tests in drugs quality control, IR spectra and DSC curves analysis, and confirmed by XRD spectrometry. Therefore, the different dissolution behavior of the test pharmaceutical products with ursodeoxycolic acid is not due to the polymorphic differences of the active ingredient.

The influence of sample preparation on IR spectra of the studied substances was investigated by comparison of spectra obtained by compression method with those by attenuated total reflectance method. The results demonstrated that there is no phase transition during sample processing.

\section{Aknowledgements}

Departament of Pharmaceutical Technology, University of Medicine and Pharmacy from Tîrgu Mureş, Romania, for providing raw materials of ibuprofen; Gedeon Richter Romania for the access to the ATR-IR equipment; joint research group of the Department of Pharmaceutical Technology, University of Medicine and Pharmacy „Iuliu Haţieganu" Cluj-Napoca, Romania, with Polipharma Industries for dissolution tests.

\section{References}

1. Patel A, Jones SA, Ferro A, Patel N. Pharmaceutical salts: a formulation trick or a clinical conundrum? Br J Cardiol. 2009;16(6):281-286.

2. Moreno-Calvo E, Calvet T, Cuevas-Diarte MA, Aquilano D. Relationship between the Crystal Structure and Morphology of Carboxylic Acid Polymorphs. Predicted and Experimental Morphologies. Cryst Growth Des. 2010;10(10):4262-4271.

3. Price SL. Predicting crystal structures of organic compounds. ChemSoc Rev. 2014;43:2098-2111.

4. Brittain HG. Polymorphism in Pharmaceutical Solids, Second Edition, Drugs and the Pharmaceutical Sciences, CRC Press. 2009.

5. ${ }^{* * *}$ DSC measurement of pharmaceuticals. Hitachi Hi-Tech TA 79. 2007 http://www.hitachi-hightech.com/file/global/pdf/products/science/appli/ ana/thermal/application_TA_079e.pdf

6. Zhang GG, Paspal SY, Suryanarayanan R, Grant DJ. Racemic species of sodium ibuprofen: characterization and polymorphic relationships. J Pharm Sci. 2003;92(7):1356-1366.

7. Dudognon E, Correia NT, Danede F, et al. Solid-Solid Transformation in Racemic Ibuprofen. Pharmaceut Res. 2013;30(1):81-89.

8. Kotar A, Kotar M, Sketa P, Plavec J. Potential of Solid-state NMR and SEM in Characterization of Tablets of Ibuprofen. Curr Pharm Anal. 2015;11(2):124-130.

9. Rossi P, Macedi E, Paoli P, et al. Solid Solid Transition between Hydrated Racemic Compound and Anhydrous Conglomerate in Na-lbuprofen: A Combined X-ray Diffraction, Solid-State NMR, Calorimetric, and Computational Study. Cryst Growth Des. 2014;14(5):2441-2452.

10. Ahuja S, Scypinski S. Handbook of Modern Pharmaceutical Analysis, 2nd Edition, Academic Press. 2010.

11. ${ }^{\star \star \star}$ International Pharmacopoeia, 4th Edition, 2013, available on http:// apps.who.int/phint/en/p/docf/

12. ${ }^{\star \star \star}$ Japanese Pharmacopoeia, 16th Edition, available on http://www. pmda.go.jp/english/pharmacopoeia/

13. Yonemochi E, Ueno $\mathrm{Y}$, Ohmae T, et al. Evaluation of amorphous ursodeoxycholic acid by thermal methods.Pharmaceut Res. 1997;14(6):798-803. 